\title{
A microfluidic-based lid device for conventional cell culture dishes to automatically control oxygen level
}

\author{
Seung Yeob Lee ${ }^{1}$ S Sung Yang ${ }^{* 1,2}$ \\ ${ }^{1}$ Department of Biomedical Science \& Engineering, Gwangju Institute of Science \& Technology, Republic of \\ Korea, ${ }^{2}$ School of Mechanical Engineering, Gwangju Institute of Science \& Technology, Republic of Korea
}

BioTechniques 64: 231-234 (May 2018) 10.2144/btn-2018-0013

Keywords: automation $\bullet$ cell $\bullet$ control $\bullet$ culture $\bullet$ dish $\bullet$ hypoxia $\bullet$ lid $\bullet$ microfluidics $\bullet$ oxygen $\bullet$ reoxygenation

Most conventional hypoxic cell culture systems undergo reoxygenation during experimental manipulations, resulting in undesirable effects including the reduction of cell viability. A lid device was developed herein for conventional cell culture dishes to resolve this limitation. The integration of multilayered microfluidic channels inside a thin membrane was designed to prevent the reoxygenation caused by reagent infusion and automatically control the oxygen level. The experimental data clearly show the reducibility of the dissolved oxygen in the infusing reagent and the controllability of the oxygen level inside the dish. The feasibility of the device for hypoxia studies was confirmed by HIF-1 $\alpha$ experiments. Therefore, the device could be used as a compact and convenient hypoxic cell culture system to prevent reoxygenation-related issues.

Recent studies showed that hypoxia plays an important role in a variety of biological conditions, including cancer and stem cells [1]. Therefore, a precise control of the in vitro hypoxic cell culture environment is important to study such hypoxia-related biological phenomena. However, conventional hypoxic cell culture devices, including a modular chamber or a gas incubator, must be opened again after hypoxic conditions have been established for experimental manipulations, such as confluency check, medium replacement and cytokine solution infusion [2]. Hypoxic cells eventually undergo reoxygenation when the culture dish is exposed to the normoxia atmosphere, resulting in reactive oxygen species generation and cell damage [3,4]. A glove box allows the manipulation of the culture dish without exposing it to the atmosphere, but it is very expensive and large; hence, it is often not available in small-scale laboratories [2]. In addition, even if the glove box is used, the reagent must be left in the glove box for a certain period or, additional operations, such as nitrogen gas bubbling, should be performed while the dissolved oxygen is removed to prevent the reoxygenation caused by the normoxic reagent infusion [5]. To overcome the inherent limits of current methods, diverse oxygen level controllable cell culture systems have been developed, including microfluidic platforms and 3D paper-based scaffolds, to generate an oxygen gradient at the closed or open microchambers [6-11]. However, most systems are not designed for use in conventional cell culture dishes, causing difficulty for them to be introduced in a laboratory setting that requires conventional culture dishes. In order to further improve their functions as well as usability in a laboratory setting, a couple of compact hypoxic cell culture devices that could be used in conventional cell culture dishes have been studied [12-15]. In spite of all these efforts, devices that can prevent reoxygenation during an experimental manipulation have not been reported yet. Moreover, all of them operate in a manner that they are inserted into the cell culture medium; hence, excluding the possibility of contamination is difficult.

Accordingly, a small-sized, low-cost culture system with the following conditions is required to resolve these limitations: 'condition 1': the device must perform the necessary function without being inserted into the culture medium inside the conventional cell culture dish to prevent contamination; 'condition 2': reoxygenation should not occur during the hypoxic cell culture because of experimental manipulations, such as reagent infusion; and 'condition 3': the oxygen level in the culture dish can be freely controlled. In this study, these conditions are achieved using an automated hypoxic culture system developed to meet all the three conditions by developing a device that incorporates microfluidic technology into the lid of conventional cell culture dishes.

To satisfy 'condition 1', the device was fabricated by stacking eight membranes (four pairs of structural and interconnecting layers) made of gas-permeable polydimethylsiloxane (PDMS) elastic polymer on the bottom of the gas-impermeable slide glass to a total thickness of $1.3 \mathrm{~mm}$, such that it could be used as the lid of a commercial $35 \mathrm{~mm}$-diameter cell culture dish. The following can be found in the PDMS membrane of the lid device: 'scavenging channel' for the oxygen-scavenging agent

\section{METHOD SUMMARY}

A microfluidic-based lid device for conventional cell culture dishes was developed for application in an in vitro hypoxia study. The lid device can reduce the dissolved oxygen in the infusing reagent and automatically control the oxygen level inside the cell culture dish. 
(sodium sulfite solution) to circulate inside the lid and go outside, 'reagent channel' for infusing a reagent (culture medium or cytokine solution) from the outside to the inside of the culture dish, and 'gas channel' for infusing gas (ambient air or nitrogen gas) directly into the dish (Figure $1 \mathrm{~A} \& \mathrm{~B}$ ).

To achieve 'condition 2', the device was designed to include a 'reagent channel' that can infuse a reagent without opening the lid when the inside of the culture dish is hypoxic. The microfluidic channels were integrated by applying the physical properties, in which the diffusion efficiently occurs in a microscale structure because of the increased surface-to-volume ratio, such that the high dissolved oxygen level corresponding to the atmospheric partial pressure of oxygen $\left(\mathrm{pO}_{2}\right)$ of the infusing reagent can be rapidly reduced [16]. This structure was designed such that the $1500 \mathrm{~mm}$-long double-layered microfluidic 'reagent channel' abuts the 'scavenging channel', where the sodium sulfite solution (oxygen-scavenging chemical reaction, $2 \mathrm{Na}_{2} \mathrm{SO}_{3}+\mathrm{O}_{2} \rightarrow 2 \mathrm{Na}_{2} \mathrm{SO}_{4}$ ) circulates through a gas-permeable thin PDMS membrane [17]. Prior to the experiment, a numerical simulation (COMSOL Multiphysics, COMSOL Inc., Burlington, MA, USA) was conducted to quantitatively estimate the reduction of dissolved oxygen. In the system, oxygen molecules are transported across different domains of heterogeneous materials including PDMS, air, infusing reagent and sodium sulfite solution. To determine the oxygen profile, the convection-diffusion and Navier-Stokes equations were solved in $3 \mathrm{D}$ simulation model with the boundary conditions including the mass flux, the continuity of $\mathrm{pO}_{2}$ governed by Henry's law, and no-slip at the channel walls $[7,16,18]$. The oxygen distribution determined by the simulation model showed oxygen reduction in the surrounding materials (PDMS, air and infusing reagent) adjacent to sodium sulfite solution. This finding is consistent with the simulation in other types of microfluidic PDMS devices using a hypoxic gas or sodium sulfite $[16,18]$. The numerical simulation and experimental studies confirmed that the device with this

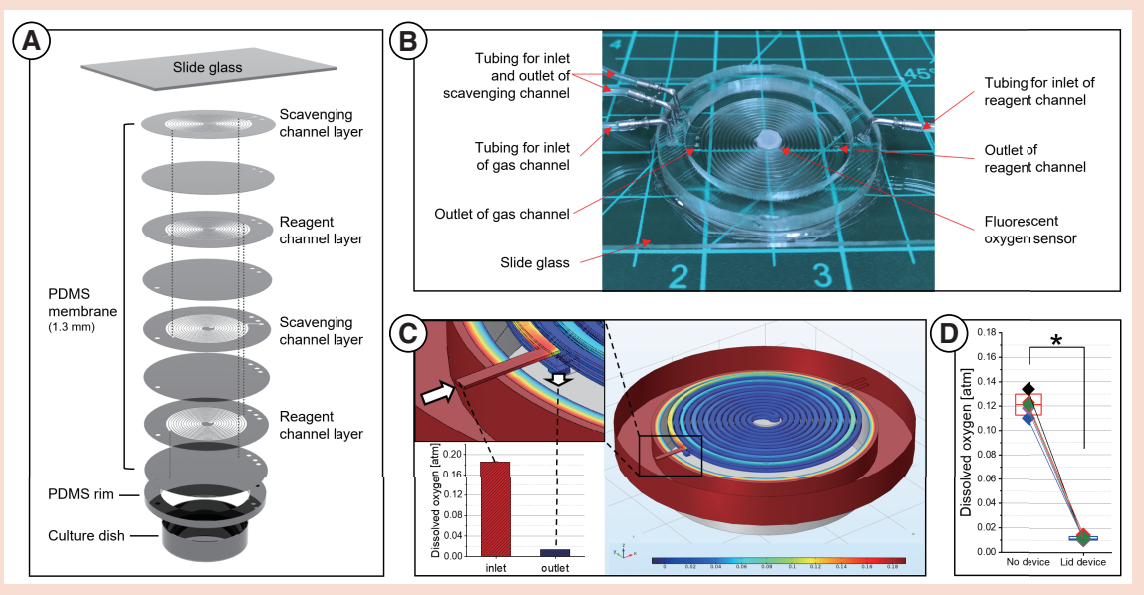

Figure 1. A schematic layout of the lid device and its numerical and experimental demonstrations showing the lid device can drastically reduce the dissolved oxygen in the infusing reagent. (A) A layout of the lid device. The eight-layers of polydimethylsiloxane (PDMS) (Sylgard 184 Silicone Elastomer, Dow Corning, Midland, MI, USA) membrane with a total thickness of $1.3 \mathrm{~mm}$ consist of four structural layers (spiral microfluidic channels with $800 \mu \mathrm{m}$ width and $200 \mu \mathrm{m}$ spacing) and four interconnecting layers connecting them through holes. The slide glass and each layer of PDMS are serially bonded by conventional oxygen plasma treatment. The dotted lines indicate the through-holes interconnecting each structural layer. (B) A photograph of the lid device. A $5 \mathrm{~mm}$-diameter film-type fluorescent oxygen sensor (VisiSens A1, PreSens GmbH, Regensburg, Germany) is attached to the center of the PDMS membrane for noninvasive sensing of the oxygen level in the culture dish. (C) A time-dependent simulation (COMSOL Multiphysics, COMSOL Inc., Burlington, MA, USA) results. A reagent with $0.186 \mathrm{~atm}$ of $\mathrm{pO}_{2}$ was infused at a flow rate of $2 \mathrm{ml} / \mathrm{min}$ for $2 \mathrm{~min}$ into the lid device, in which the oxygen-scavenging agent (sodium sulfite) circulates at a flow rate of $1 \mathrm{ml} / \mathrm{h}$. The $\mathrm{pO}_{2}$ calculated every $1 \mathrm{~s}$ at the outlet of the reagent channel is less than $0.014 \mathrm{~atm}(0.0138 \pm 0.0001 \mathrm{~atm}$, mean $\pm \mathrm{SD})$, which means that more reagent volume than the recommended culture medium volume $(2 \mathrm{ml})$ of the $35 \mathrm{~mm}$-diameter dish can be hypoxic during the infusion. (D) Wilcoxon signed rank test result for the presence of the lid device on the dissolved oxygen reduction (reagent infusion at a flow rate of $2 \mathrm{ml} / \mathrm{min}$ while the sodium sulfite circulates at a flow rate of $1 \mathrm{ml} / \mathrm{h}$ ). The $\mathrm{pO}_{2}$ of the infused reagent without the lid is $0.121 \pm 0.008 \mathrm{~atm}$, on the other hand, the one with the lid device is $0.012 \pm 0.001 \mathrm{~atm}\left({ }^{*} \mathrm{p}<0.05\right.$ at five tests of paired samples). The lid device can reduce the dissolved oxygen level of the infused reagent compared with routine procedures, such as pipetting, more than $90 \%$.

SD: Standard deviation.

structure can reduce the dissolved oxygen of the infused reagent by more than $90 \%$ (Figure 1C \& D).

To realize 'condition 3', the lid device was designed for sodium sulfite solution to circulate inside the device through the 'scavenging channel'. Nitrogen gas (0 atm $\mathrm{pO}_{2}$ of $99.999 \%$ purified $\mathrm{N}_{2}$ tank) and ambient air $\left(0.186\right.$ atm $\mathrm{pO}_{2}$ of $37^{\circ} \mathrm{C}$ humidified $5 \% \mathrm{CO}_{2}$ incubator) were directly infused into the dish through the 'gas channel'. A simple proportional-integral-derivative algorithm has been applied to automate oxygen level control and measure $\mathrm{pO}_{2}$ in the dish through an oxygen sensor. The infusion of sodium sulfite, nitrogen gas and ambient air was controlled through commercially available syringe pumps and regulators (Figure 2A). As shown in Figure 2B (a representative control result), the measured $\mathrm{pO}_{2}$ exhibited a rapid change rate (average $0.043 \mathrm{~atm} / \mathrm{min}$ ) and a stable maintenance (average 1.88\% coefficient of variation) and faithfully followed the preset target $\mathrm{pO}_{2}$ value in the algorithm.

The HIF-1 $\alpha$ induction experiment, which is a major cellular mediator to hypoxia, was performed for the feasibility study of the microfluidic-based lid device for the hypoxic cell culture [19]. The experimental results using the lid device showed that the HIF-1 $\alpha$ level of the cultured cells under hypoxic condition $\left(\mathrm{pO}_{2}\right.$ at 0.047 and 0 atm) was higher $(\mathrm{p}<0.05)$ than that of normoxic condition ( $\mathrm{pO}_{2}$ at $\left.0.186 \mathrm{~atm}\right)$. This is consistent with the previous studies that showed that $\mathrm{HIF-1} \alpha$, which is continuously synthesized in cells, is rapidly degraded by the ubiquitin-proteasome system under normoxic conditions mediated by the prolyl hydroxylase enzymes [20]. Meanwhile, the degradation is prevented under hypoxic conditions [21]. Furthermore, the level of $\mathrm{HIF-1} \alpha$ (ranging from 0.75 to 2.88 ) gradually increased as $\mathrm{pO}_{2}$ decreased (ranging from 0.186 to $0 \mathrm{~atm})$, which is also concordant with the inverse proportion of $\mathrm{HIF}-1 \alpha$ to oxygen levels observed in other types of hypoxic culture systems (Figure 2C) [7,13] Therefore, the hypoxic culture using the device does not disturb the natural cellular response to hypoxia, and is applicable to hypoxia-related studies, including HIF-1 $\alpha$ and its downstream cascade.

Compared with previous compact devices compatible with conventional cell culture dishes [12-15], the lid device could provide a capability of not only reducing the dissolved oxygen in infusing reagent, 

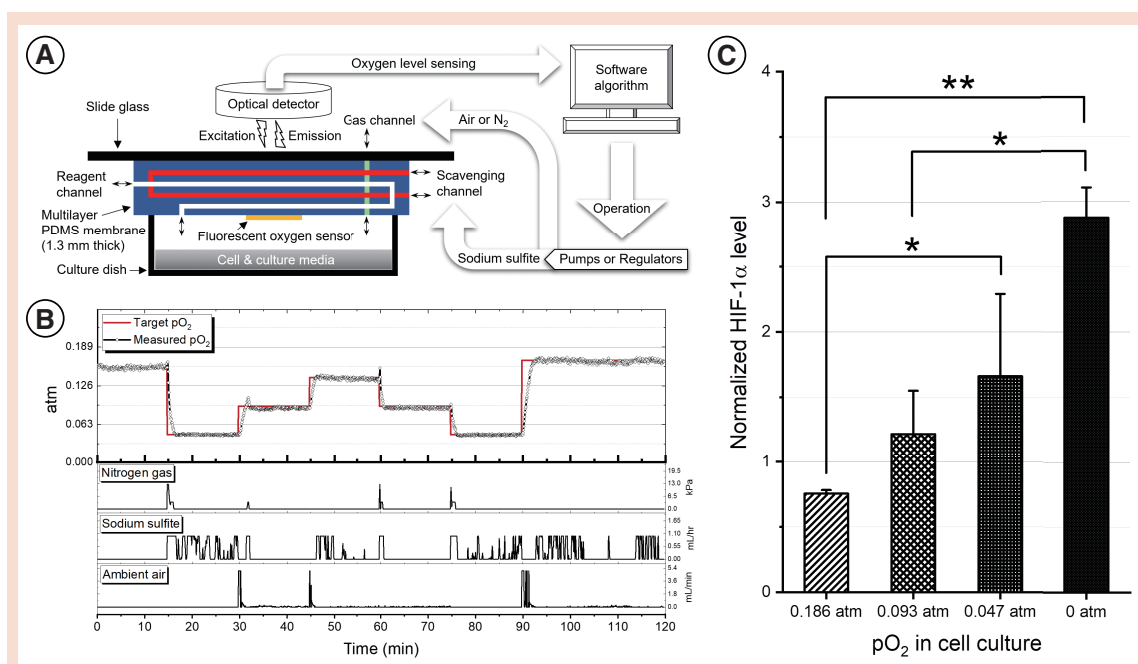

Figure 2. A schematic diagram of the working principle of the lid device and the experimental demonstration of HIF-1 $\alpha$ induction study as well as its performance on the automatic oxygen level control. (A) Diagram showing the cross-section view of the lid device and the operating procedure for the automatic oxygen level control. The $\mathrm{pO}_{2}$ level measured by an optical detector placed on top of the transparent lid device is transmitted to a computer, where the automatic oxygen level control program based on a PID algorithm has been installed (LabVIEW, National Instruments Corporation, Austin, TX, USA). The amount of sodium sulfite, nitrogen gas and ambient air infused into the lid device to control the oxygen level inside the dish is calculated by the automatic oxygen level control program, and the pumps and the regulators are automatically actuated as well. (B) A representative example demonstrating how precisely the automatic oxygen level control system works. The algorithm was preset to sequentially control the target $\mathrm{pO}_{2}$ at $0.047,0.093,0.140,0.093$ and $0.047 \mathrm{~atm}$ for 15 min each and $0.167 \mathrm{~atm}$ for the last 30 min after the initial 15 min oxygen-uncontrolled period (red line). As shown in the measured $\mathrm{pO}_{2}$ of the culture dish, the nitrogen gas injection is only used to accelerate the oxygen reduction rate. The constant oxygen level can be (gas tank-free) achieved only by the injection of sodium sulfite and ambient air. (C) The total HIF-1 $\alpha$ concentration (HIF-1 $\alpha$ human ELISA Kit, Thermo Fisher Scientific, Waltham, MA, USA) in the cultured cells measured under various $\mathrm{pO}_{2}(0.186,0.093,0.047$ and $0 \mathrm{~atm}$ ) with the lid device equipped. The culture conditions were as follows: the human cell line (NCI-H1975, ATCC, Manassas, VA, USA) was cultured in an RPMI-1640 medium (Sigma-Aldrich, St. Louis, MO, USA) supplemented with 10\% fetal bovine serum (Atlas Biologicals, Fort Collins, CO, USA) for $4 \mathrm{~h}$ in a $37^{\circ} \mathrm{C}$ humidified $5 \% \mathrm{CO}_{2}$ incubator. After removing the lid device from the culture dish, a mixture of cell lysis buffer (10X, \#9803, Cell Signaling Technology, Danvers, MA, USA) and protease/ phosphatase inhibitor cocktail (100X, \#5872, Cell Signaling Technology) was immediately added (within a few seconds) to obtain the cell lysates. The total HIF-1 $\alpha$ was measured in the cell lysates according to the manufacturer's guideline using HIF-1 $\alpha$ human ELISA Kit (Thermo Fisher Scientific). The simultaneously measured HIF-1 $\alpha$ of the cells incubated with a commercial lid (Corning Inc., Corning, NY, USA) in normoxia and total protein concentration (bicinchoninic acid protein assay kit, Thermo Fisher Scientific) was used as control samples to normalize the HIF-1 $\alpha$ concentration. Each experiment was independently performed three-times. The normalized HIF-1 $\alpha$ levels (mean \pm SD) are $0.75 \pm 0.02,1.22 \pm 0.31$, $1.66 \pm 0.58$ and $2.88 \pm 0.26$ for each target $\mathrm{pO}_{2}$. The difference is statistically significant $\left({ }^{*} \mathrm{p}<0.05\right.$; $* * p<0.005$; Kruskal-Wallis test followed by Tukey's post hoc analysis).

PID: Proportional-integral-derivative; SD: Standard deviation.

but also precise controlling of oxygen level without direct contact between device and culture media leading to a low possibility of contamination. Moreover, the lid device also provides a freedom of choice in materials (chemical agent or gas) for oxygen control. Thus, for example, the lid device could be used for hyperoxia-related studies because it can use high-oxygen-content gases or oxygen-generating agents [22]. In addition, this device could be used in studies requiring the change of the oxygen level during cell culture, including the ischemia-reperfusion injury model, because the oxygen level can be arbitrarily controlled [23]. Furthermore, the lid device is compact ( $50 \mathrm{~mm}$ in diameter) and it is expected to be affordable with cost as low as $\$ 3000$ for the entire system, including the lid device (a couple of dollars) and all other components (pump, regulator and oxygen sensor). Therefore, the lid device system reported in this work is a promising alternative to a large and expensive (up to $\$ 50,000$ ) conventional glove box to prevent reoxygenation-related issues during hypoxic cell culture.

\section{Author contributions}

SY Lee performed the experiments and wrote the paper. S Yang supervised the study and corrected the manuscript.

\section{Financial \& competing interests disclosure}

This study was supported by the National Research Foundation of Korea (NRF) grant funded by the Korean government (MSIP) (NRF-2016M3A7B4910556). The authors have no other relevant affiliations or financial involvement with any organization or entity with a financial interest in or financial conflict with the subject matter or materials discussed in the manuscript apart from those disclosed.

No writing assistance was utilized in the production of this manuscript.

\section{Open access}

This work is licensed under the Creative Commons Attribution 4.0 License. To view a copy of this license, visit http://creativecommons.org/licenses/by/4.0/

\section{References}

1. Mohyeldin A, Garzon-Muvdi T, QuinonesHinojosa A. Oxygen in stem cell biology: a critical component of the stem cell niche. Cell Stem Cell 7(2), 150-161 (2010)

2. Wang $\mathrm{RX}$, Jin FS, Zhong $\mathrm{H}$. A novel experimental hypoxia chamber for cell culture. Am. J. Cancer Res. 4(1), 53-60 (2014).

3. Zhang $\mathrm{K}$, Zhao $\mathrm{T}$, Huang $X$ Dissolved oxygen concentration in the medium during cell culture: defects and improvements. Cell Biol. Int. 40(3), 354-360 (2016).

4. Zhang YM, Liao H, Zhong SP Effect of N-n-butyl haloperidol iodide on ROS/JNK/Egr-1 signaling in $\mathrm{H} 9 \mathrm{c} 2$ cells after hypoxia/reoxygenation. Sci. Rep. 5, 11809 (2015).

5. Wenger RH, Kurtcuoglu V, Scholz CC, Marti $\mathrm{HH}$, Hoogewijs D. Frequently asked questions in hypoxia research. Hypoxia (Auckl.) 3, 35-43 (2015).

6. Brennan MD, Rexius-Hall ML, Elgass LJ, Eddington DT. Oxygen control with microfluidics. Lab Chip 14(22), 4305-4318 (2014).

7. Byrne MB, Leslie MT, Patel HS, Gaskins HR, Kenis PJA. Design considerations for open-wel microfluidic platforms for hypoxic cell studies. Biomicrofluidics 11(5), 054116 (2017).

8. Chang CW, Cheng YJ, Tu M A polydimethylsiloxane-polycarbonate hybrid microfluidic device capable of generating perpendicular chemical and oxygen gradients for cell culture studies. Lab Chip 14(19), 3762-3772 (2014).

9. Derda R, Laromaine A, Mammoto A Papersupported 3D cell culture for tissue-based bioassays. Proc. Natl Acad. Sci. USA 106(44), 18457-18462 (2009)

10. Boyce MW, Kenney RM, Truong AS, Lockett MR. Quantifying oxygen in paper-based cell cultures with luminescent thin film sensors. Anal. Bioanal. Chem. 408(11), 2985-2992 (2016).

11. Mehta G, Mehta K, Sud D Quantitative measurement and control of oxygen levels in microfluidic poly(dimethylsiloxane) bioreactors 
during cell culture. Biomed. Microdevices 9(2), 123-134 (2007).

12. Oppegard SC, Nam KH, Carr JR, Skaalure SC, Eddington DT. Modulating temporal and spatial oxygenation over adherent cellular cultures. PLOS ONE 4(9), e6891 (2009).

13. Li CY, Chaung W, Mozayan C, Chabra R, Wang P, Narayan RK. A new approach for on-demand generation of various oxygen tensions for in vitro hypoxia models. PLOS ONE 11(5), e0155921 (2016).

14. Brennan MD, Rexius-Hall ML, Eddington DT. A 3D-printed oxygen control insert for a 24-well plate. PLoS ONE 10(9), e0137631 (2015).

15. Huang $Y$, Zitta K, Bein B, Steinfath M, Albrecht M. An insert-based enzymatic cell culture system to rapidly and reversibly induce hypoxia: investigations of hypoxia-induced cell damage, protein expression and phosphorylation in neuronal IMR-32 cells. Dis. Model. Mech. 6(6), 15071514 (2013).

16. Kim MC, Lam RHW, Thorsen T, Asada HH. Mathematical analysis of oxygen transfer through polydimethylsiloxane membrane between double layers of cell culture channel and gas chamber in microfluidic oxygenator. Microfluid. Nanofluid. 15(3), 285-296 (2013).

17. Zhao B, Li Y, Tong HL Study on the reaction rate of sulfite oxidation with cobalt ion catalyst. Chem. Eng. Sci. 60(3), 863-868 (2005).

18. Wang L, Liu WM, Wang YL Construction of oxygen and chemical concentration gradients in a single microfluidic device for studying tumor cell-drug interactions in a dynamic hypoxia microenvironment. Lab Chip 13(4), 695-705 (2013).

19. Semenza GL. HIF-1: mediator of physiological and pathophysiological responses to hypoxia. J. Appl. Physiol. 88(4), 1474-1480 (2000).

20. Bruick RK, Mcknight SL. A conserved family of prolyl-4-hydroxylases that modify HIF. Science 294(5545), 1337-1340 (2001).

21. Li QF, Wang XR, Yang YW, Lin H. Hypoxia upregulates hypoxia inducible factor (HIF)-3 alpha expression in lung epithelial cells: character- ization and comparison with $\mathrm{HIF-1}$ alpha. Cell Res. 16(6), 548-558 (2006).

22. Feldmeier J, Carl U, Hartmann K, Sminia P. Hyperbaric oxygen: does it promote growth or recurrence of malignancy? Undersea Hyperb. Med. 30(1), 1-18 (2003).

23. Eltzschig HK, Eckle T. Ischemia and reperfusionfrom mechanism to translation. Nat. Med. 17(11), 1391-1401 (2011).

Received 24 January 2018; Accepted for publication: 2 March 2018

Address correspondence to: Sung Yang, Department of Biomedical Science \& Engineering, Gwangju Institute of Science \& Technology, Republic of Korea, Tel.: +82 62715 2407; Fax: +82 62715 2384; E-mail: syang@ gist.ac.kr

To purchase reprints of this article, contact s.cavana@future-science.com

\section{how accurate can you actually be?}

If you're in pain while pipetting

\section{"Ovation pipettes are very easy to use. My hands never get tired or hurt."}

\section{V

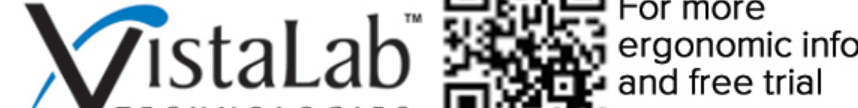 tiennologites}

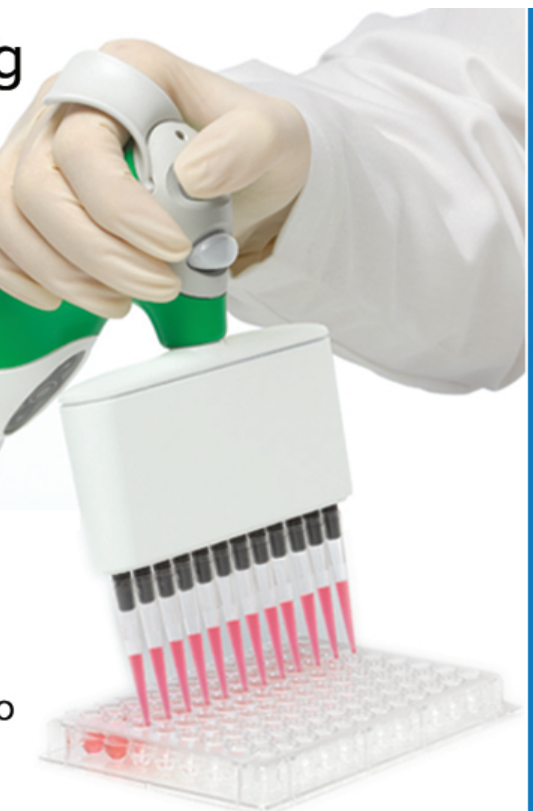

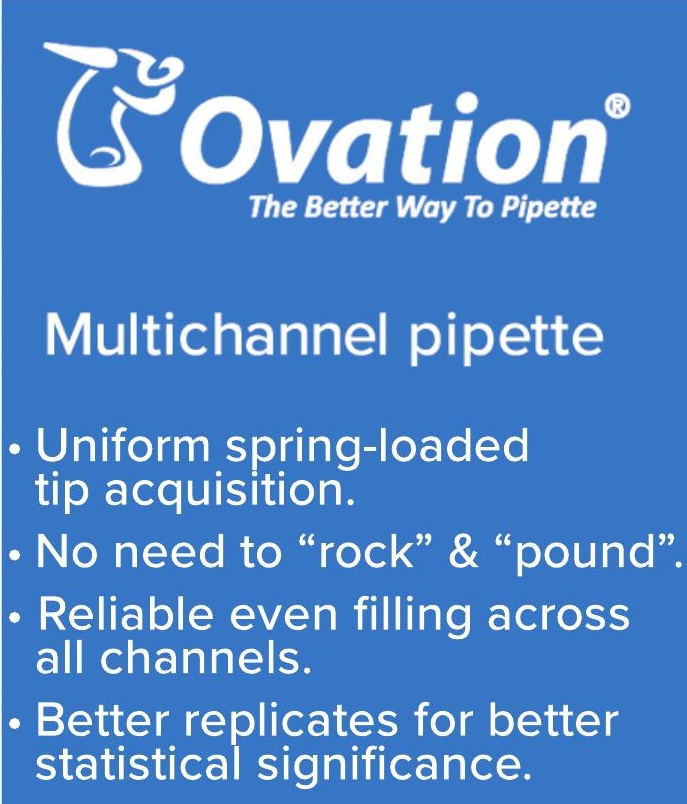

